Research Article

\title{
Fault Detection of Reciprocating Compressor Valve Based on One-Dimensional Convolutional Neural Network
}

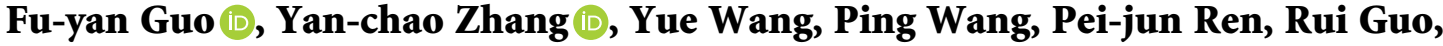 \\ and Xin-Yi Wang
}

Tianjin Chengjian University, Tianjin 300384, China

Correspondence should be addressed to Fu-yan Guo; happytime76@163.com

Received 30 December 2019; Revised 12 March 2020; Accepted 15 April 2020; Published 29 April 2020

Academic Editor: Cedrick A. K. Kwuimy

Copyright (C) $2020 \mathrm{Fu}$-yan Guo et al. This is an open access article distributed under the Creative Commons Attribution License, which permits unrestricted use, distribution, and reproduction in any medium, provided the original work is properly cited.

\begin{abstract}
Reciprocating compressors are important equipment in oil and gas industries which closely relate with the healthy development of the enterprise. It is essential to detect the valve fault because valve failures account for $60 \%$ in total failures. For this field, an artificial neural network (ANN) is widely used, but a complex network is not suitable for its low accuracy and easy overfitting. This paper proposes a fault diagnosis model of a reciprocating compressor valve based on a one-dimensional convolutional neural network (1DCNN). This method takes the differential pressure and differential temperature of each compressor stage as the input of $1 \mathrm{DCNN}$, using the characteristics of the CNN to extract the features and finally using Softmax to classify the fault. In order to verify this method, it is compared with LM-BP, RBF, and BP neural networks. The results show that the fault recognition rate of $1 \mathrm{DCNN}$ reaches $100 \%$, which proves the effectiveness and feasibility of the proposed method.
\end{abstract}

\section{Introduction}

Reciprocating compressor is one of the most widely used compressor technologies in today's oil and gas industries. It can compress a variety of gases and has a wide range of applications, high compression efficiency, and stable working pressure. However, due to its complicated structure and many vulnerable parts, once a failure cannot be detected and eliminated in time, it will bring huge losses to the enterprise [1-7]. The literature indicates that $60 \%$ of reciprocating compressor failures are valve failures, and the number of shutdowns caused by valve failures accounted for $36 \%$ and accounted for $50 \%$ of the total maintenance costs [8-10]. Therefore, monitoring the failure of the reciprocating compressor valve can not only reduce the overall maintenance cost but also improve the stability of the compressor operation.

Due to the characteristic of deep learning [11] which can overcome the shortcomings of traditional learning model networks such as complexity, low accuracy, and easy overfitting, it has quickly become a hot topic in the research field in the past few years. It has made breakthrough progress in image recognition, voice recognition, language processing, and other fields. In view of the achievements of deep learning in other fields, it has also received more and more attention in the fault diagnosis and intelligent identification of modern industrial machinery and equipment. Tran et al. [12] proposed a hybrid deep belief network. The hybrid algorithm was pretrained by the deep belief network, and the training results were classified by the SfAM. The single and combined faults of the suction and exhaust valves were diagnosed by acquiring the three signals of vibration, pressure, and current of the reciprocating compressor. Liu et al. [13] proposed an intelligent fault diagnosis method combining local mean decomposition (LMD) and stack noise reduction automatic encoder (SDAE) to classify faults of reciprocating compressor gas valves. The results show that the classification accuracy of this method can reach $92.72 \%$. Zhao et al. [14] proposed a compound interpolation envelope LMD (CIE-LMD) algorithm to analyze the vibration signals of the reciprocating compressor in different states. The results show that this method can effectively diagnose the fault of excessive bearing clearance of reciprocating compressors. 
A convolutional neural network [15], as one of the classic algorithms in deep learning, is the first learning algorithm that can truly successfully train multilayer networks. Its special convolutional layer and pooling layer can effectively reduce the complexity of the network, greatly reduce the number of free parameters during training, and have strong robustness and fault tolerance. The entire network is easy to train and optimize. These characteristics of convolutional neural networks have attracted a large number of scholars to study their theory and applications. Some experts have also tried to use convolutional neural networks to solve the problem of fault diagnosis. Yang et al. [16] used three sensors to collect vibration signals when the reciprocating compressor valve fails, and directly used as the input of the convolutional neural network to make full use of its characteristics that automatically extract characteristic signals, and then carried out fault diagnosis and obtained higher fault recognition rate. Ince et al. [17] used the one-dimensional motor current signal as the input of a convolutional neural network and then diagnosed the fault of the motor. The fault recognition rate is as high as $97.8 \%$. Zhang et al. [18] extracted vibration signals during diesel engine fire faults, and automatically extracted fault features through one-dimensional convolutional neural networks, and then classified the faults. The results proved that the accuracy rate under different operating conditions can reach $100 \%$.

At present, vibration signals are mostly used as sample data for fault diagnosis of reciprocating compressor valves. However, according to the thermal performance parameter fault diagnosis method of reciprocating compressors established by Qi [19], it is known that the most direct response of a gas valve when a leak occurs is the pressure ratio imbalance, and the temperature and pressure responses are the most obvious [20-24]. As mentioned earlier, the CNNs' ability to learn to extract the optimal features and the system can achieve the optimal fault classification and fault detection accuracy through proper training. Therefore, this paper takes the differential pressure and differential temperature of each stage of the multistage reciprocating compressor as sample data. A fault diagnosis model of a reciprocating compressor valve based on one-dimensional convolutional neural network is established, the features in the sample data are automatically extracted, and then fault classification is performed by the Softmax function. The advantages of this method are (1) directly using the temperature signal and pressure signal can well achieve online fault diagnosis and improve the quality and efficiency of detection and (2) automatically extracting the fault characteristics of the reciprocating compressor valve through the model, without the need to manually extract the characteristics and selection, which improves the accuracy of fault diagnosis.

\section{Structure and Principle of CNN}

Convolutional neural networks are a typical type of deep neural networks. They are supervised learning algorithms such as BP neural networks, so they need to be equipped with labeled data as training samples. The essence is to add multiple filters before the traditional fully connected network. The input data are convolved and pooled layer by layer to achieve the effect of extracting input features [25-27]. The biggest improvement of the convolutional neural network lies in the convolutional layer, which greatly reduces the number of parameters, so as to greatly reduce the calculation amount of the network.

A typical CNN topology is shown in Figure 1. The input of the network is a $2 \mathrm{D}$ feature map. After that, each convolution layer and pooling layer alternately appear, and the output of this layer is used as the input of the next layer, and so on. At the end of the $\mathrm{CNN}$, several fully connected layers are generally used to link each neuron in the previous layer. This process can expand the feature map into a one-dimensional vector for final classification.

CNN convolutional layers are used to extract local features from the input neuron data. Each type of convolution corresponds to an extracted feature. The same type of convolution kernel has the property of weight sharing, which can be learned in parallel and greatly reduces the free parameters. And the complexity of the network is reduced to a certain extent. The feature information extracted by each type of convolution kernel is limited, so multiple convolution kernels are generally used to extract feature information. The input of the convolutional neural network is $X$, and $H^{l}$ represents the feature maps $\left(H^{0}=X\right)$ of the $l$ th layer, assuming that $H_{j}^{l}$ is the $j$ th feature map of the convolutional layer of the lth layer, and the process of $H_{j}^{l}$ generation is as follows:

$$
H_{j}^{l}=f\left(\sum_{i=1}^{k} H_{i}^{l-1} \times W_{i j}^{(l)}+b_{j}^{l}\right),
$$

where $W_{i j}^{(l)}$ represents the weight matrix connecting the $i$ th feature map of layers $l-1$ and the $j$ th feature map of the layer $l ; k$ represents the number of feature maps in layers $l-1 ; i$ and $j$ are the indexes of the input and output feature maps; and $b_{j}^{l}$ is the offset corresponding to each feature map of the layer $l$. Finally, the $j$ th feature map $H_{j}^{l}$ of the layer $l$ is obtained by the excitation function $f(\cdot)$. The excitation function can be the ReLU function, sigmoid function, tanh $(x)$ function, radial basis function, etc.

The CNN pooling layer is also called the downsampling layer, and its role is to scale and map the data graph of the previous layer, so as to reduce the data dimension. Suppose $H_{j}^{l}$ is the $j$ th feature map of the $l$ th pooling layer. The generation process is as shown in equation (2), where down (.) represents a pooling function, and each output feature map corresponds to its own multiplicative bias $\beta_{j}^{l}$ and an additive bias $b_{j}^{l}$ :

$$
H_{j}^{l}=f\left(\beta_{j}^{l} \operatorname{down}\left(H_{j}^{l-1}\right)+b_{j}^{l}\right) .
$$

High-level features are extracted by sequentially stacking convolutional layers and pooling layers, and these features are flattened into one-dimensional vectors. For example, the feature $H_{j}^{P_{2}}$ extracted from $P_{2}$ in Figure 1 is flattened into a one-dimensional feature vector which is the output of the fully connected layer $F_{1}$. Similar to the multilayer neural network thereafter, the output of the $F_{2}$ layer is as follows: 


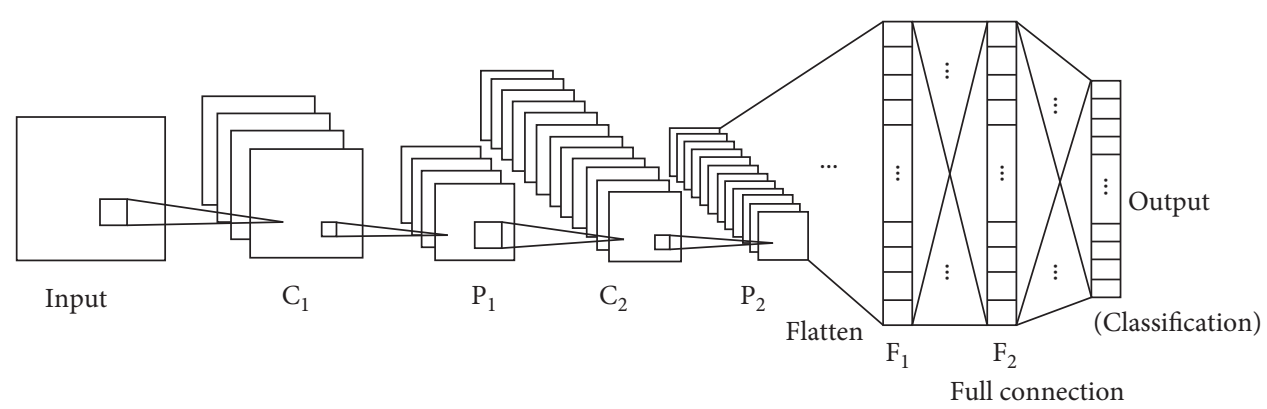

Figure 1: Topology of a typical CNN.

$$
H_{j}^{F_{2}}=f\left(\sum_{i=1}^{k} H_{i}^{F_{1}} \times W_{i j}^{\left(F_{2}\right)}+b_{j}^{F_{2}}\right),
$$

where $H_{i}^{F_{1}}=$ flatten $\left(H_{j}^{P_{2}}\right)$ is an flattened one-dimensional vector; $k$ represents the number of feature maps in the layer $F_{1}$; and $W_{i j}^{\left(F_{2}\right)}$ and $b_{j}^{F_{2}}$ represent the tied weights and the tied bias of the layer $F_{2}$.

At present, convolutional neural networks are mostly a supervised deep learning model. Network training is similar to traditional artificial neural networks. In the fully connected layer, the back propagation algorithm (BP) is still used to optimize the parameters to reduce the error to the expected value. The parameters are optimized by the gradient descent method.

\section{Experimental Study}

3.1. Test Rig and Instrumentation. The experimental bench consists of a six-stage water-cooled reciprocating compressor and a synchronous motor of the type TK2800-20/ 2150 , with a maximum speed of $300 \mathrm{r} / \mathrm{min}$. The compressor consists of six cylinders, the pressure of the suction gas is 1961.33 Pa (gauge pressure), and the final exhaust pressure after compression of each cylinder is $31.4 \mathrm{MPa}$ (gauge pressure). When the multistage reciprocating compressor gas valve leaks, the hot gas will blow back into the cylinder. This will not only heat the intake gas during the intake process but also continue to heat the gas at the beginning of the compression process, causing the exhaust temperature to rise. The change in temperature will cause the pressure to change, and the change in the previous stage will cause the pressure and heat to increase in the next stage of gas compression. Due to design reasons, the cooling device is fixed at the exhaust port of the cylinder, so the heat generated during the compression of the gas cannot be removed in time, which causes the volume of the gas to expand, the quality of the compressed gas to decrease, and ultimately the efficiency of the compressor to decline. In summary, when the valve leaks, the temperature and pressure of the gas entering and exiting the cylinder are directly affected. Therefore, this experiment uses 12 pressure sensors and 12 temperature sensors to collect the temperature and pressure of the gas at the inlet and outlet of each cylinder. The sensor installation diagram is shown in Figure 2. In the figure, $P_{1}$ and $T_{1}$ represent the sensors for collecting gas pressure and temperature at the inlet of the cylinder $I, P_{2}$ and $T_{2}$ represent the sensors for collecting gas pressure and temperature at the outlet of the cylinder $I$, and the sensors required for other cylinders are installed similarly.

3.2. Experimental Procedure. The experiment first obtains the data that are collected by all sensors when the valve of each cylinder is normal and then calculates the differential pressure and differential temperature between the inlet and outlet gases of each cylinder. For example, if the pressure and temperature of the inlet gas of the cylinder $I$ are $P_{1}$ and $T_{1}$ and the pressure and temperature of the outlet gas are $P_{2}$ and $T_{2}$, then the differential pressure and differential temperature between the inlet and outlet gases of the first-stage cylinder are $P_{\mathrm{d} 1}$ and $T_{\mathrm{d} 1}$. Because it is a 6-stage reciprocating compressor, 12 numbers are obtained after all calculations. These 12 numbers form a set of sample data. Table 1 shows the symbols of the differential pressure and differential temperature between the inlet and outlet gases of each cylinder. Next, the above experiments were repeated under the same operating conditions but simulated six types of real faults, first-stage valve leak, second-stage valve leak, thirdstage valve leak, fourth-stage valve leak, fifth-stage valve leak, and sixth-stage valve leak. Table 2 is the fault code table.

\section{Established 1DCNN Fault Diagnosis Model}

Unlike image data, temperature and pressure data are one dimensional, and it is more reasonable to use the one-dimensional convolutional CNN to process these data. In this paper, the feature extraction and fault diagnosis of reciprocating compressor valves are combined, and a one-dimensional convolutional neural network (1DCNN) model is proposed. As shown in Figure 3, the model uses the original temperature and pressure signals without the need for manual feature extraction. The classic VGG model replaces the convolution kernel size with $3^{*} 3$ and the pooling kernel size with $2 * 2$, which can greatly reduce the number of parameters and improve the accuracy of the model, while maintaining the spatial structure of the input of the previous layer. Because the input data of the model established in this paper are $12 * 1$ (the 6-stage reciprocating compressor and the differential pressure and differential temperature between the gas inlet and outlet of each stage), the size of the convolution kernel in this model is set to $3^{*} 1$ and the size of the pooling kernel is set to $2 * 1$. The Keras framework is used 


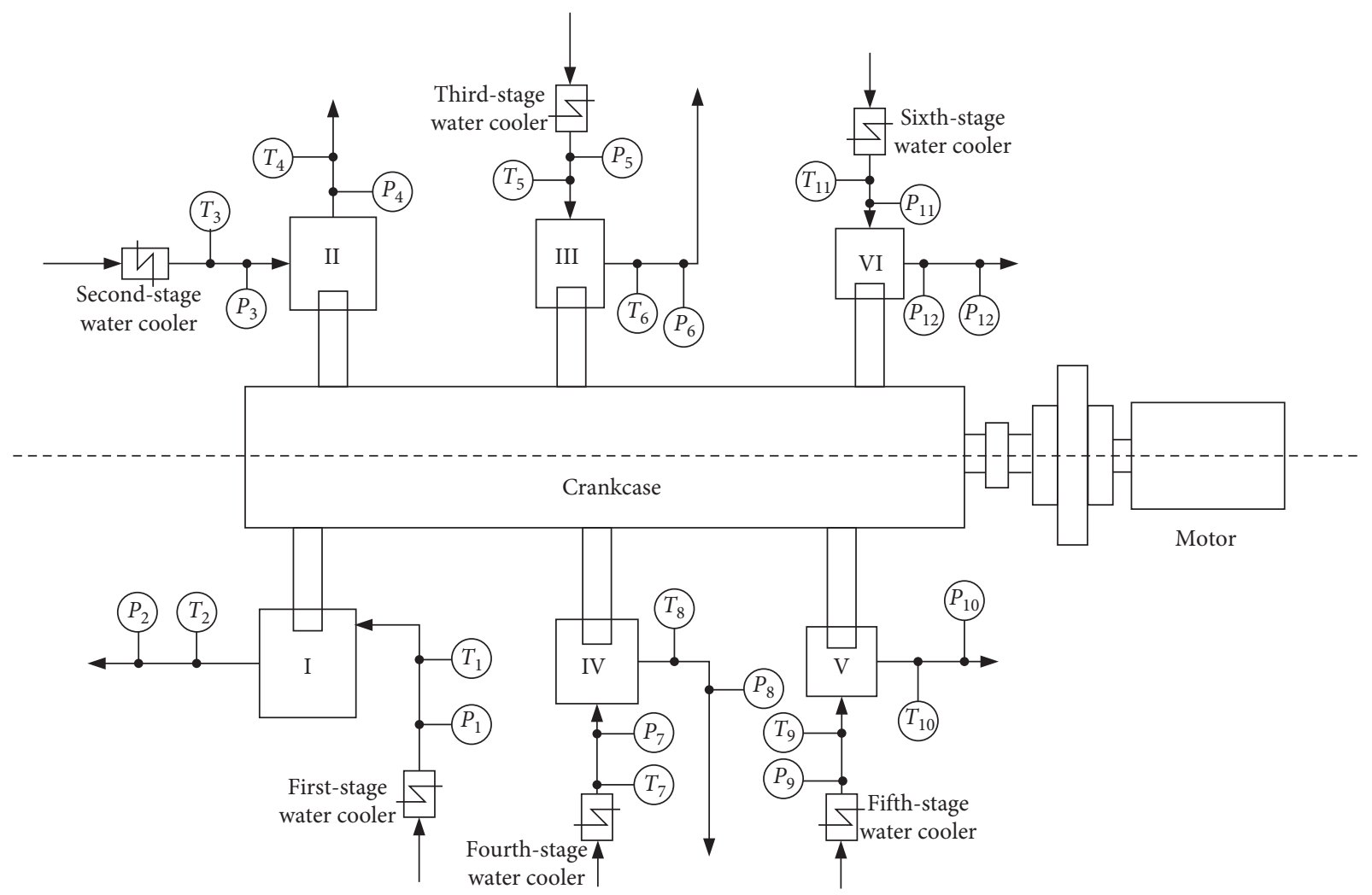

Figure 2: Experimental sensor installation diagram.

TABLE 1: Symbolic table of the differential pressure and differential temperature between the inlet and outlet gases at all stages.

\begin{tabular}{lcc}
\hline Cylinder & Differential pressure (Mpa) & Differential temperature $\left({ }^{\circ} \mathrm{C}\right)$ \\
\hline First-stage cylinder & $P_{\mathrm{d} 1}$ & $T_{\mathrm{d} 1}$ \\
Second-stage cylinder & $P_{\mathrm{d} 2}$ & $T_{\mathrm{d} 2}$ \\
Third-stage cylinder & $P_{\mathrm{d} 3}$ & $T_{\mathrm{d} 3}$ \\
Fourth-stage cylinder & $P_{\mathrm{d} 4}$ & $T_{\mathrm{d} 4}$ \\
Fifth-stage cylinder & $P_{\mathrm{d} 5}$ & $T_{\mathrm{d} 5}$ \\
Sixth-stage cylinder & $P_{\mathrm{d} 6}$ & $T_{\mathrm{d} 6}$ \\
\hline
\end{tabular}

TABLE 2: The fault code table.

\begin{tabular}{|c|c|c|c|}
\hline Number & Operating status & $\begin{array}{c}\text { Fault } \\
\text { number }\end{array}$ & $\begin{array}{c}\text { Expected } \\
\text { output }\end{array}$ \\
\hline 1 & Normal status & 0 & $(1000000)$ \\
\hline 2 & First-stage valve leak & 1 & $(0100000)$ \\
\hline 3 & $\begin{array}{l}\text { Second-stage valve } \\
\text { leak }\end{array}$ & 2 & $(0010000)$ \\
\hline 4 & Third-stage valve leak & 3 & $(0001000)$ \\
\hline 5 & $\begin{array}{c}\text { Fourth-stage valve } \\
\text { leak }\end{array}$ & 4 & $(0000100)$ \\
\hline 6 & Fifth-stage valve leak & 5 & $(0000010)$ \\
\hline 7 & Sixth-stage valve leak & 6 & $(0000001)$ \\
\hline
\end{tabular}

to construct a training model in Python. After several verifications and adjustments, the $1 \mathrm{DCNN}$ model for reciprocating compressor valve fault diagnosis uses the network structure shown in Table 3, including 2 convolutional layers, 2 maximum pooling layers, 1 flatten layer, and 2 fully connected layers whose length is 128 and 64, respectively.
Finally, the Softmax function is used to classify the 7 faults in Table 2. In order to overcome the disappearance of gradients and speed up network training, the activation functions of each layer use the ReLU function.

The 1DCNN model in this paper uses a multiclass crossentropy function to measure the Softmax classification results. $p(x)$ represents the target class probability distribution, $q(x)$ represents the predicted probability distribution, and $p(x)$ and $q(x)$ cross-entropy function formula is as follows:

$$
H(p, q)=-\sum_{x} p(x) \log (q(x))
$$

In order to minimize the loss function, this article uses the Adam algorithm proposed by Kingma et al. [28] to optimize the gradient descent method. Its advantage lies in the design of independent adaptive learning rates for different parameters when calculating the gradient, and basically only very few parameters need to be adjusted. 


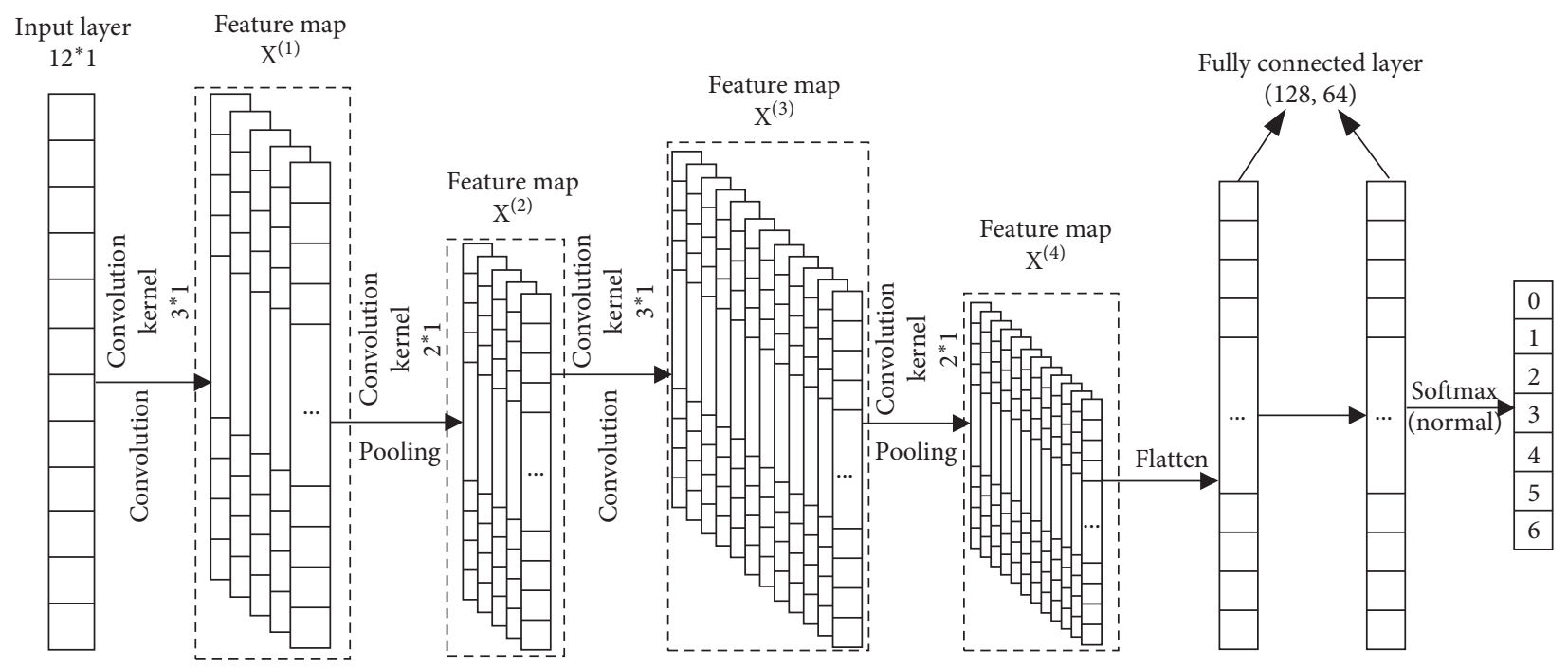

FIGURE 3: Structure of the 1DCNN model.

TABle 3: Parameters of the 1DCNN model.

\begin{tabular}{lccc}
\hline Layer (type) & Number of convolution kernels & Output shape & Parameter \\
\hline Input layer & 32 & $12^{*} 1$ & 0 \\
Conv1 (Conv1D) & 32 & $10^{*} 1^{*} 32$ & 128 \\
Activation ReLU & & $10^{*} 1^{*} 32$ & 0 \\
MaxPool1 & 64 & $5^{*} 1^{*} 32$ & 0 \\
Conv2 (Conv1D) & & $3^{*} 1^{*} 64$ & 6208 \\
Activation ReLU & & $3^{*} 1^{*} 64$ & 0 \\
MaxPool2 & $2^{*} 1^{*} 64$ & 0 \\
FC1/flatten & 128 & 0 \\
FC2 & & 64 & 8256 \\
Softmax & & 7 & 455 \\
\hline
\end{tabular}

1DCNN diagnosis flowchart is shown in Figure 4. The differential pressure and differential temperature data of each stage of gas when a certain level of valve failure is collected are divided into a training set and a test set. The training set data are trained by the $1 \mathrm{DCNN}$ to perform forward and back propagation to minimize errors. The $1 \mathrm{DCNN}$ training model that meets the requirements uses the test set data for evaluation and verification.

\section{Test and Verification}

5.1. Description of the Data Set. Through the experimental process described in Section 3.2, a total of 2100 sets of sample data are obtained. Each set of data is 12 numbers representing the differential pressure and differential temperature between the inlet and outlet gases of each cylinder. Table 4 shows some sample data. According to the holdout method [29], the data are decomposed and recombined: $70 \%$ are used as training data, including 1470 data samples; 30\% are used as test samples, including 630 data samples. The training samples are used for model establishment, and the test samples are used for model evaluation.
5.2. Experimental Results and Discussion. $70 \%$ of the sample data are randomly selected as the input for network training, and the parameters are adjusted and updated after forward and back propagation. The remaining $30 \%$ of the sample data are used to test the fault recognition performance of the trained model, and the accuracy curve and loss curve are shown in Figure 5. The red dashed line in the loss curve represents that the set error is $1 e^{-5}$. In order to prevent the random factors from affecting the training results, 10 experiments are repeated. The results are shown in Figure 6. The accuracy of each diagnosis is stable at $100 \%$.

The comparison methods include the Levenberg-Marquardt neural network (LM-BP), radical basis function neural network (RBF), and conventional BP neural network [30]. Statistics and analysis of the training results are shown in Table 5, and Figure 7 is a comparison of the results of the fault diagnosis test. When using the 1DCNN model for training, the required error is reached when the number of iterations reaches 23 , the average test error is 0.0001 , and the test accuracy rate reaches the highest level of $100 \%$. LM-BP achieves the required error after 87 iterations. The test error and test accuracy are slightly lower than the 


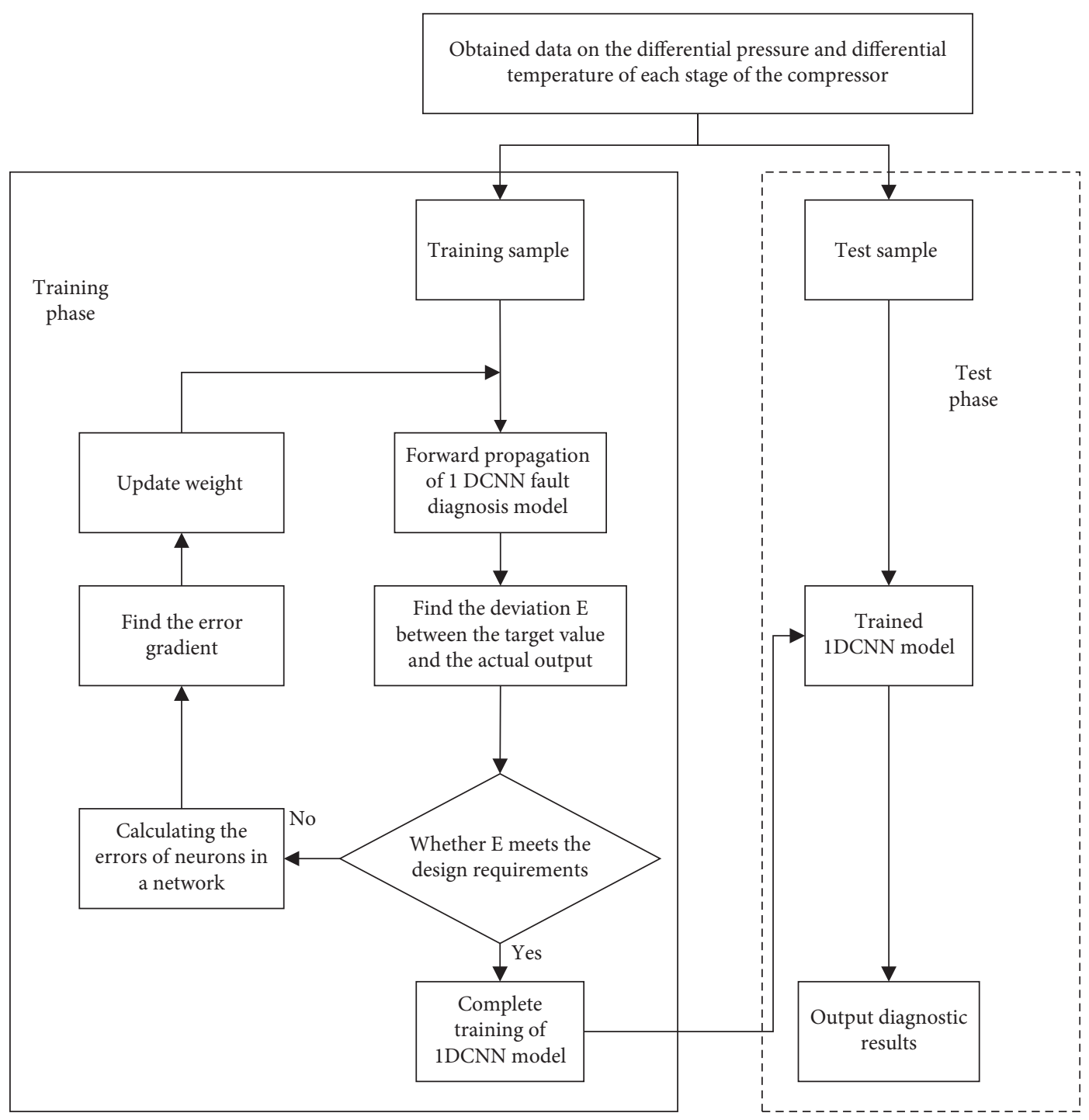

Figure 4: Flowchart of 1DCNN fault diagnosis.

TABle 4: Part of the training sample.

\begin{tabular}{lcccccccccccc}
\hline Fault number & $\mathrm{P}_{\mathrm{d} 1}$ & $\mathrm{P}_{\mathrm{d} 2}$ & $\mathrm{P}_{\mathrm{d} 3}$ & $\mathrm{P}_{\mathrm{d} 4}$ & $\mathrm{P}_{\mathrm{d} 5}$ & $\mathrm{P}_{\mathrm{d} 6}$ & $\mathrm{~T}_{\mathrm{d} 1}$ & $\mathrm{~T}_{\mathrm{d} 2}$ & $\mathrm{~T}_{\mathrm{d} 3}$ & $\mathrm{~T}_{\mathrm{d} 4}$ & $\mathrm{~T}_{\mathrm{d} 5}$ & $\mathrm{~T}_{\mathrm{d} 6}$ \\
\hline 0 & 0.213 & 0.6 & 1.05 & 2.73 & 6.5 & 11.6 & 78 & 87 & 67 & 99 & 96 & 70 \\
0 & 0.236 & 0.61 & 0.94 & 2.78 & 6.8 & 12.3 & 81 & 119 & 58 & 101 & 93 & 74 \\
1 & 0.133 & 0.7 & 1.09 & 3.14 & 6.4 & 11.4 & 76 & 91 & 77 & 101 & 90 & 70 \\
1 & 0.154 & 0.67 & 1.11 & 2.68 & 6.4 & 11.2 & 94 & 126 & 80 & 104 & 87 & 64 \\
2 & 0.26 & 0.52 & 0.97 & 2.85 & 6.4 & 11 & 11 & 94 & 60 & 95 & 96 & 70 \\
2 & 0.309 & 0.57 & 1.08 & 3 & 6 & 12.1 & 96 & 97 & 74 & 107 & 85 & 78 \\
3 & 0.226 & 0.78 & 0.83 & 3 & 6.4 & 10.8 & 77 & 119 & 88 & 101 & 91 \\
3 & 0.232 & 0.75 & 0.95 & 3.05 & 6.5 & 11.5 & 83 & 107 & 77 & 100 & 89 & 70 \\
4 & 0.212 & 0.6 & 1.27 & 2.82 & 6.4 & 10.7 & 78 & 86 & 70 & 99 & 97 & 70 \\
4 & 0.236 & 0.63 & 1.02 & 2.99 & 6.5 & 12.5 & 86 & 78 & 80 & 89 & 95 \\
5 & 0.214 & 0.61 & 0.97 & 3.4 & 6.5 & 12.6 & 95 & 97 & 71 & 120 & 98 \\
5 & 0.212 & 0.62 & 0.98 & 3.2 & 6.3 & 11.1 & 81 & 89 & 71 & 124 & 92 \\
6 & 0.203 & 0.62 & 0.96 & 3 & 7.4 & 13 & 92 & 93 & 73 & 105 & 101 & 106 \\
6 & 0.203 & 0.66 & 1.02 & 2.97 & 6.4 & 7.6 & 88 & 96 & 73 & 106 & 97 & 84 \\
\hline
\end{tabular}




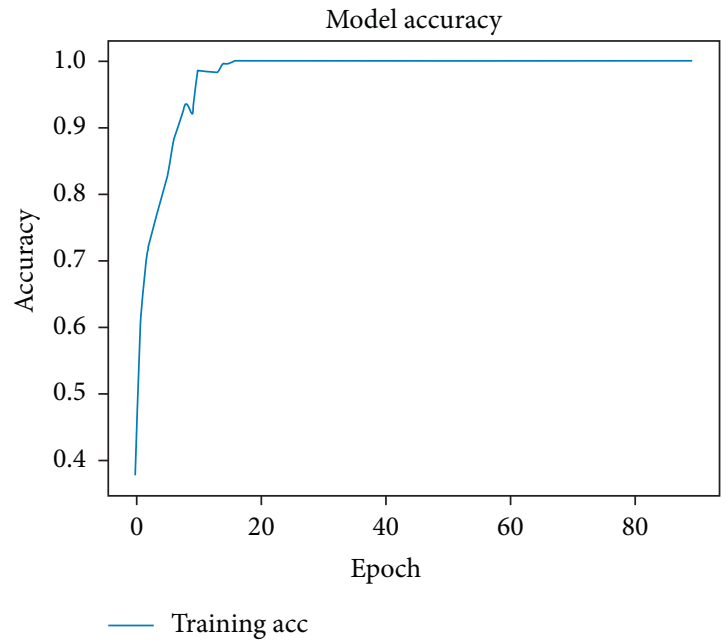

(a)

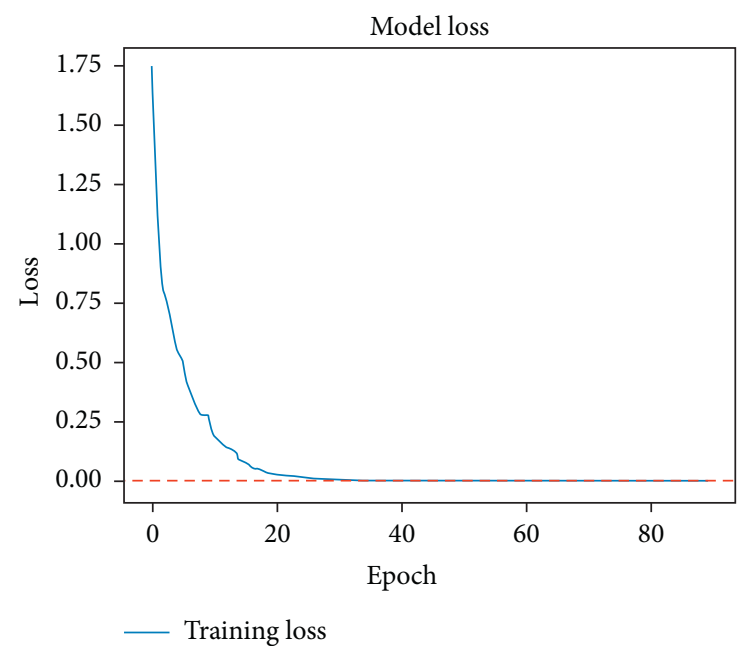

(b)

Figure 5: Accuracy curve and loss curve obtained by training with this method.

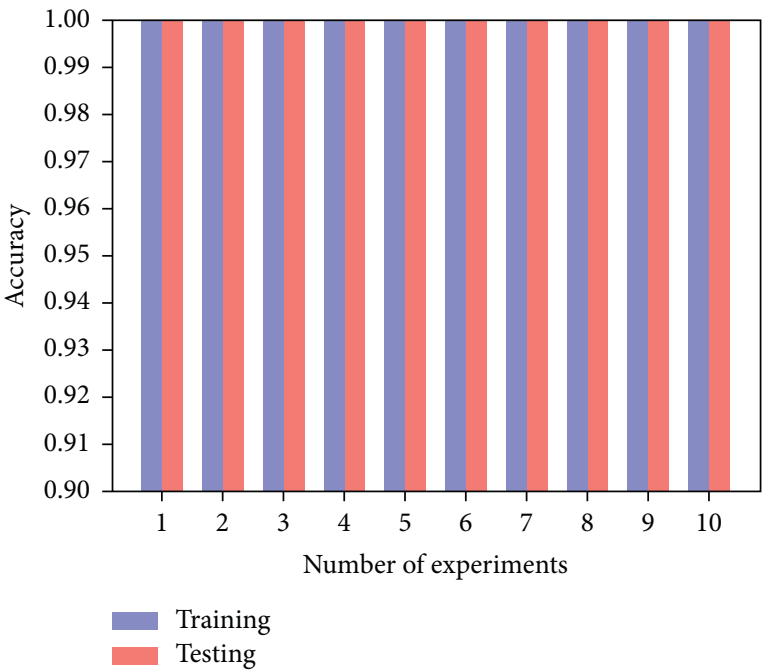

Figure 6: Ten diagnostic results on the data set using this method.

$1 \mathrm{DCNN}$ model. The average test accuracy is $96.78 \%$, and the average test error is 0.001 . Although the RBF method and the $\mathrm{BP}$ method do not reach the required error, the test accuracy also reaches a high level. The average test accuracy of the RBF method is $92.25 \%$, and the average test error is 0.005 . The average test accuracy of the BP method is $89.72 \%$, and the average test error is 0.064 . The above results show that the method proposed in this paper can not only have high diagnostic accuracy but also the diagnostic results are very stable.

In order to further verify the diagnostic performance of the 1DCNN model, the proportion of training samples and test samples in the data is adjusted, and the proportions of training samples are set to $70 \%, 50 \%$, and $30 \%$. The best three
TABLE 5: Comparison of training results using different methods.

\begin{tabular}{lcccc}
\hline Method & 1DCNN & LM-BP & RBF & BP \\
\hline Number of iterations & 23 & 87 & 100 & 198 \\
Training error & $1 e^{-5}$ & $1 e^{-5}$ & 0.00089 & 0.0947 \\
\hline
\end{tabular}

sets of results are selected from the training results of these three sample sets, and the loss curve and accuracy curve of the three sets of training results are compared, as shown in Figure 8 . The results show that, whether it is a loss curve or an accuracy curve, $70 \%$ of the training data converge the fastest, followed by $50 \%$ of the training data, and $30 \%$ of the training data are the slowest.

5.3. Visualization of Learning Features. The advantage of $1 \mathrm{DCNN}$ is that it can automatically extract the features of the sample data for training. In order to verify the feature extraction capability of this model, the $\mathrm{t}$-distributed stochastic neighbor embedding ( $t-S N E$ ) method is used to reduce the high-dimensional data to two-dimensional data and visualize. Because the output layer of the $1 D C N N$ model is Softmax classification, the fully connected layer of the previous layer is used as the sample feature extracted by the model, and the 64-dimensional feature is reduced to twodimensional feature and visualized by the t-SNE method. Figure 9 shows a visualization of the features extracted using the 1DCNN model. The colored numbers in the figure correspond to the fault numbers in Table 2. It is obvious that each fault condition is well aggregated, and the different characteristics are effectively separated. The above results show that the 1DCNN model has excellent feature extraction capabilities and can adaptively extract features required for fault diagnosis. 


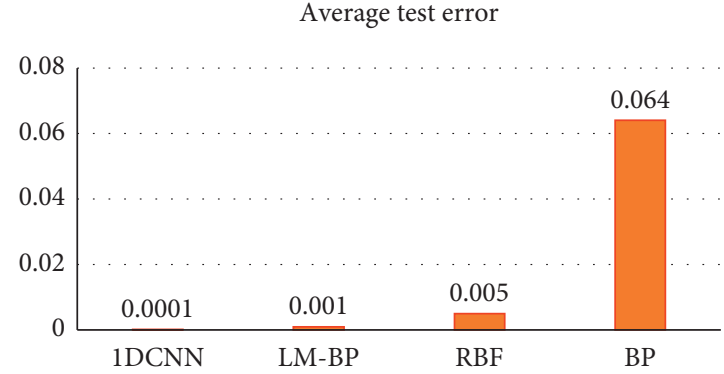

(a)

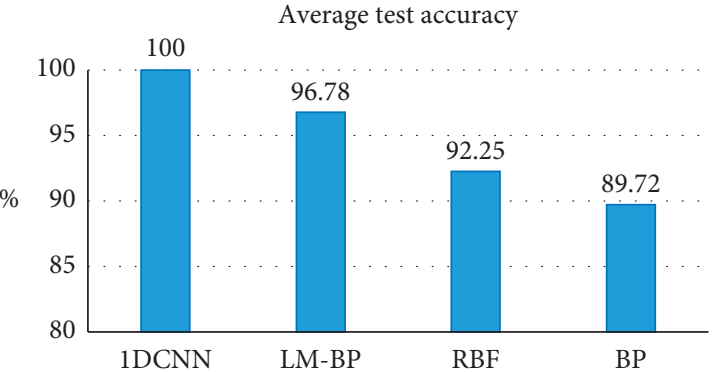

(b)

Figure 7: Diagnostic results of different methods.

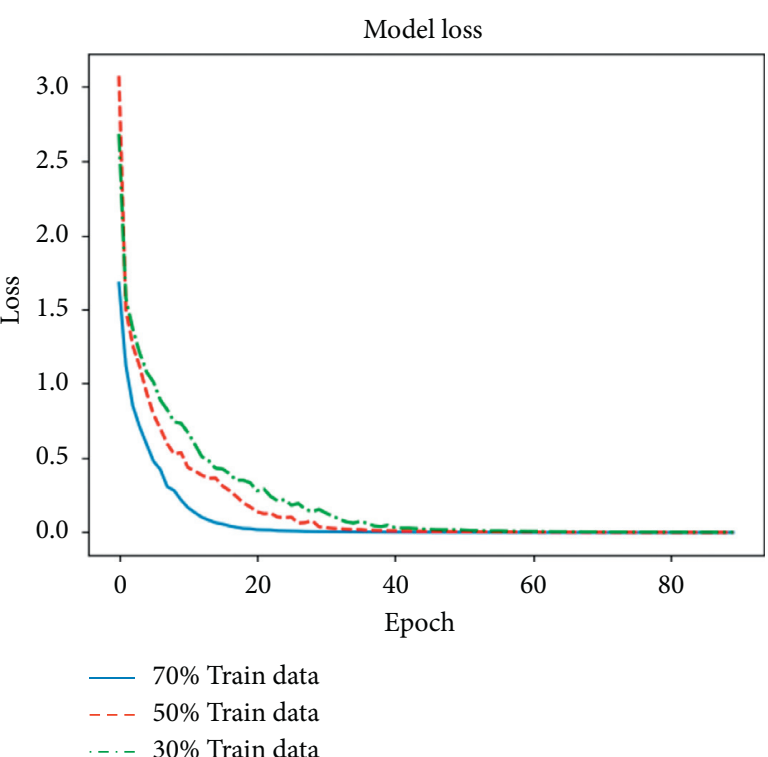

(a)

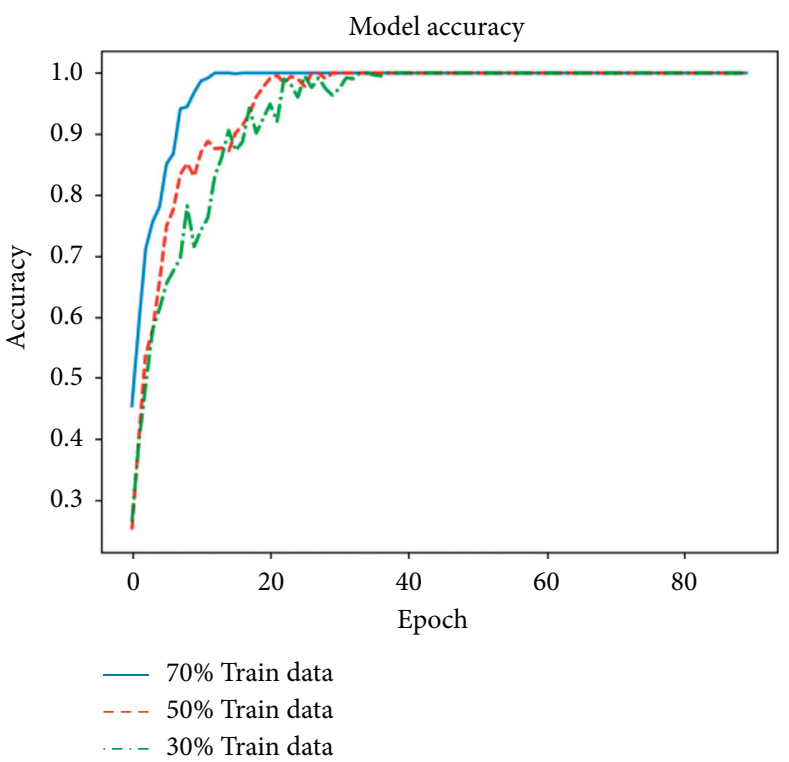

(b)

FIGURE 8: Loss curve and accuracy curve for different sample proportions.

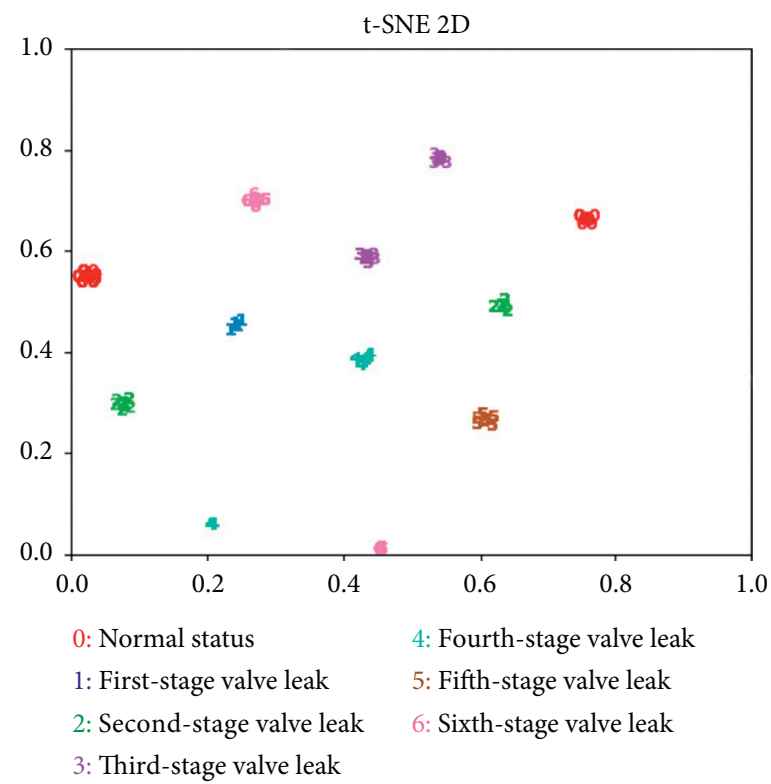

Figure 9: Feature visualization based on the t-SNE method. 


\section{Conclusions}

This paper proposes a one-dimensional convolutional neural network-based fault diagnosis method for reciprocating a compressor gas valve. Two types of data such as the differential pressure and differential temperature between each stage of a multistage reciprocating compressor are tested. And compared with the fault classification results of $\mathrm{BP}$ neural network, RBF neural network, and LM-BP neural network, the experimental results show that the fault recognition rate of the BP neural network reaches $89.72 \%$, and the test error is 0.064 ; the fault recognition rate of the $\mathrm{RBF}$ neural network is $92.25 \%$, and the test error is 0.005 ; the fault recognition rate of the LM-BP neural network is $96.78 \%$, and the test error is stable at 0.001 ; and the $1 \mathrm{DCNN}$ fault recognition rate reaches $100 \%$, and the test error is stable at 0.0001 . By comparison, it is concluded that the accuracy of the $1 \mathrm{DCNN}$ is higher, which can effectively monitor the operating conditions of the reciprocating compressor valve, thereby reducing or even avoiding accidents. At present, when establishing a model in this paper, it is necessary to continuously adjust the parameters manually. In the future, I hope that a universal model can appear.

\section{Data Availability}

The data used to support the findings of this study are available from the corresponding author upon request.

\section{Conflicts of Interest}

The authors declare that they have no conflicts of interest.

\section{Acknowledgments}

This research was funded by the Tianjin Natural Science Foundation. The project name is "Development of Energy Management and Fault Pre-Diagnosis System for Industrial System Equipment" (project number 17JCTPJC52200).

\section{References}

[1] M. Elhaj, F. Gu, A. D. Ball, A. Albarbar, M. Al-Qattan, and A. Naid, "Numerical simulation and experimental study of a two-stage reciprocating compressor for condition monitoring," Mechanical Systems and Signal Processing, vol. 22, no. 2, pp. 374-389, 2008.

[2] Y. Tang, Q. Liu, J. Jing, Y. Yang, and Z. Zou, “A framework for identification of maintenance significant items in reliability centered maintenance," Energy, vol. 118, pp. 1295-1303, 2017.

[3] H. Y. Zhao, M. Q. Xu, J. D. Wang, and Y. B. Li, "A Parameters optimization method for planar joint clearance model and its application for dynamics simulation of reciprocating compressor," Journal of Sound and Vibration, vol. 334, no. 5, pp. 416-433, 2015.

[4] H. Y. Zhao, M. Q. Xu, and J. D. Wang, "Local mean decomposition based on rational hermite interpolation and its application for fault diagnosis of reciprocating compressor," Journal of Mechanical Engineering, vol. 51, no. 1, pp. 83-89, 2015.
[5] S. M. Ali, K. H. Hui, L. M. Hee et al., "Automated valve fault detection based on acoustic emission parameters and support vector machine," Alexandria Engineering Journal, vol. 57, no. 1, pp. 491-498, 2017.

[6] H. Cui, L. Zhang, R. Kang, and X. Lan, "Research on fault diagnosis for reciprocating compressor valve using information entropy and SVM method," Journal of Loss Prevention in the Process Industries, vol. 22, no. 6, pp. 864-867, 2009.

[7] J. Ma, Z. N. Jiang, and J. J. Gao, "Feature extraction method based on chaotic fractal theory and its application in fault diagnosis of gas valves," Journal of Vibration and Shock, vol. 31, no. 19, pp. 26-30, 2012.

[8] P. Loukopoulos, G. Zolkiewski, I. Bennett et al., "Abrupt fault remaining useful life estimation using measurements from a reciprocating compressor valve failure," Mechanical Systems and Signal Processing, vol. 121, pp. 359-372, 2019.

[9] H. P. Bloch, "A Practical Guide to Compressor Technology," Chemical Engineering Progress, vol. 114, no. 1, pp. 8-9, 2007.

[10] J. R. Kolodziej and J. N. Trout, "An image-based pattern recognition approach to condition monitoring of reciprocating compressor valves," Journal of Vibration and Control, vol. 24, no. 19, pp. 4433-4448, 2018.

[11] G. E. Hinton, S. Osindero, and Y.-W. Teh, "A fast learning algorithm for deep belief nets," Neural Computation, vol. 18, no. 7, pp. 1527-1554, 2006.

[12] V. T. Tran, F. AlThobiani, T. Tinga, A. Ball, and G. Niu, "Single and combined fault diagnosis of reciprocating compressor valves using a hybrid deep belief network," Proceedings of the Institution of Mechanical Engineers, Part C: Journal of Mechanical Engineering Science, vol. 232, no. 20, pp. 3767-3780, 2018.

[13] Y. Liu, L. X. Duan, Z. Yuan et al., "An intelligent fault diagnosis method for reciprocating compressors based on LMD and SDAE," Sensors, vol. 19, no. 5, p. 1041, 2019.

[14] H. Y. Zhao, J. D. Wang, L. Jay et al., "A compound interpolation envelope local mean decomposition and its application for fault diagnosis of reciprocating compressors," Mechanical Systems and Signal Processing, vol. 110, pp. 273-295, 2018.

[15] Y. Lecun, L. Bottou, Y. Bengio, and P. Haffner, "Gradientbased learning applied to document recognition," Proceedings of the IEEE, vol. 86, no. 11, pp. 2278-2324, 1998.

[16] H.-b. Yang, J.-a. Zhang, L.-1. Chen, H.-l. Zhang, and S.-l. Liu, "Fault diagnosis of reciprocating compressor based on convolutional neural networks with multisource raw vibration signals," Mathematical Problems in Engineering, vol. 2019, Article ID 6921975, 7 pages, 2019.

[17] T. Ince, S. Kiranyaz, L. Eren, M. Askar, and M. Gabbouj, "Real-time motor fault detection by 1-D convolutional neural networks," IEEE Transactions on Industrial Electronics, vol. 63, no. 11, pp. 7067-7075, 2016.

[18] K. Zhang, J. F. Tao, C. J. Qin et al., "Fault diagnosis of diesel engine misfire based on random discard and batch standardized deep convolutional neural network," Journal of Xi'an Jiaotong University, vol. 53, no. 8, pp. 159-166, 2019.

[19] W. M. Qi, "A method for judging thermal failure of reciprocating compressors," Mechanical and Electrical Equipment, vol. 5, pp. 32-34, 2002.

[20] P. Loukopoulos, G. Zolkiewski, I. Bennett et al., "Reciprocating compressor prognostics of an instantaneous failure mode utilizing temperature only measurements," Applied Acoustics, vol. 147, pp. 77-86, 2019.

[21] G. D. Dang, Fault Diagnosis and Failure Analysis of Reciprocating Compressor Gas Valve, Xi'an Shiyou University, Xi'an, China, 2018. 
[22] M. Farzaneh-Gord, A. Niazmand, M. Deymi-Dashtebayaz, and H. R. Rahbari, "Effects of natural gas compositions on CNG (compressed natural gas) reciprocating compressors performance," Energy, vol. 90, pp. 1152-1162, 2015.

[23] M. Farzaneh-Gord and H. Khoshnazar, "Valve fault detection for single-stage reciprocating compressors," Journal of Natural Gas Science and Engineering, vol. 35, pp. 1239-1248, 2016.

[24] J. Townsend, M. A. Badar, and J. Szekerces, "Updating temperature monitoring on reciprocating compressor connecting rods to improve reliability," Engineering Science and Technology, an International Journal, vol. 19, no. 1, pp. 566573, 2016.

[25] A. Krizhevsky, I. Sutskever, and G. Hinton, "ImageNet classification with deep convolutional neural networks," Communications of the ACM, vol. 25, pp. 1097-1105, 2012.

[26] O. Abdeljaber, O. Avci, S. Kiranyaz, M. Gabbouj, and D. J. Inman, "Real-time vibration-based structural damage detection using one-dimensional convolutional neural networks," Journal of Sound and Vibration, vol. 388, pp. 154-170, 2017.

[27] J. An, P. Ai, S. Xu et al., "An intelligent fault diagnosis method for rotating machinery based on one-dimensional convolutional neural network," Journal of Nanjing University (Natural Science), vol. 55, no. 1, pp. 133-142, 2019.

[28] D. P. Kingma and J. L. Ba, "Adam: A Method for Stochastic Optimization,” Computer Science, vol. 8, pp. 2333-9721, 2015.

[29] C. Dwork, V. Feldman, M. Hardt, T. Pitassi, O. Reingold, and A. Roth, "The reusable holdout: preserving validity in adaptive data analysis," Science, vol. 349, no. 6248, pp. 636-638, 2015.

[30] H. Zhang, Research on Fault Diagnosis of Multi-Stage Reciprocating Compressor Air Valve Based on LM-BP Neural Network, Donghua University, Shanghai, China, 2015. 